\title{
An Unusual Case of Acquired Uterine Arteriovenous Malformation With Persistent Scar Ectopic Pregnancy Successfully Managed With Uterine Artery Embolization
}

\author{
Geetha Visvalingam ${ }^{\mathrm{a}, \mathrm{c}}$, Ryan Wai Kheong Lee ${ }^{\mathrm{a}}$, Tse Yeun Tan ${ }^{\mathrm{b}}$, \\ Heng Hao Tan ${ }^{\mathrm{b}}$
}

\begin{abstract}
Uterine arteriovenous malformation (AVM) is a rare entity in gynecology, with fewer than 100 cases reported in the literature. AVM is an abnormal arteriovenous connection lacking an intervening capillary network on histopathological examination. We report an unusual case of acquired AVM after dilatation and curettage of cesarean scar ectopic pregnancy managed with uterine artery embolization and concurrent methotrexate administration for persistently elevated betahuman chorionic gonadotropin levels.
\end{abstract}

Keywords: Arteriovenous; Malformations; Scar; Ectopic; Embolization

\section{Introduction}

Uterine arteriovenous malformation (AVM) is a rare entity in gynecology, with fewer than 100 cases reported in the literature since Dubreuil and Loubat reported the first case of AVM in 1926 [1]. Uterine AVM is an abnormal arteriovenous connection lacking an intervening capillary network on histopathological examination. AVMs are high-flow systems, with blood coursing through to the venous system under arterial pressure and flow. The venous system is unable to accommodate the high-pressure, high-flow state, resulting in a higher propensity for bleeding. It can be a rare cause of massive uterine bleeding. It usually presents with intractable torrential or intermittent bleeding, suggestive of arterial source and may be life-threatening.

Uterine AVMs can be congenital or acquired. Acquired

Manuscript accepted for publication March 04, 2016

aDepartment of Obstetrics \& Gynecology, KK Women and Children's Hospital, 100 Bukit Timah Road, Singapore 229899, Singapore

bDepartment of Reproductive Medicine, KK Women and Children's Hospital, 100 Bukit Timah Road, Singapore 229899, Singapore

${ }^{\mathrm{c} C o r r e s p o n d i n g ~ A u t h o r: ~ G e e t h a ~ V i s v a l i n g a m, ~ D e p a r t m e n t ~ o f ~ O b s t e t r i c s ~ \& ~ G y-~}$ necology, KK Women and Children's Hospital, 100 Bukit Timah Road, Singapore 229899, Singapore. Email: gvisvalingam@gmail.com

doi: http://dx.doi.org/10.14740/jmc2458w
AVMs are usually secondary to uterine trauma such as dilatation and curettage, therapeutic abortion, uterine surgery, intrauterine devices, neoplasia and gestational trophoblastic disease [2].

We report an unusual case of acquired AVM after dilation and curettage of cesarean scar ectopic pregnancy managed with uterine artery embolization (UAE) with concurrent methotrexate administration for persistently elevated beta human chorionic gonadotropin (hCG) levels.

\section{Case Report}

A 41-year-old female with past history of two previous lower segment cesarean sections (LSCSs) was diagnosed with scar ectopic pregnancy at a private hospital for which she underwent uterine dilation and curettage at 6 weeks gestation. No histology was available. The procedure was complicated by heavy vaginal bleeding and the patient developed a small cervical hematoma which was managed conservatively. She was discharged 1 week after surgical evacuation. After discharge, she had persistent heavy vaginal bleeding for a month before presenting to our tertiary hospital emergency room for further assessment.

On presentation, she was clinically stable and was admitted for further investigations. Her initial full blood count showed hemoglobin of 12.3 on admission with normal coagulation profile. Urine hCG was positive and serum beta hCG was elevated at $628 \mathrm{IU} / \mathrm{L}$.

A transvaginal ltrasound pelvis showed endometrium to be $4 \mathrm{~mm}$ and a $4.2 \times 3.1 \times 2.7 \mathrm{~cm}$ complex cystic structure containing multiple tubular serpentine cystic spaces at the lower anterior wall of the uterus close to the site of the previous LSCS scar (Fig. 1). Color Doppler showed marked increased vascularity within these cystic spaces. In view of the patient's recent dilatation and curettage, findings were suspicious of uterine AVM.

A multiplanar, multisequential enhanced magnetic resonance imaging (MRI) pelvis was then performed which showed a rounded, complex T2W hyperintense and TW1 hypointense structure containing blood products and avidly enhancing 2.6 $\mathrm{cm}$ complex structure after contrast, at the site of the previous LSCS scar in the lower anterior wall of the uterus (Fig. 2). Correlation with the ultrasound study revealed that this may represent a post-treatment AVM. No active extravasation of 


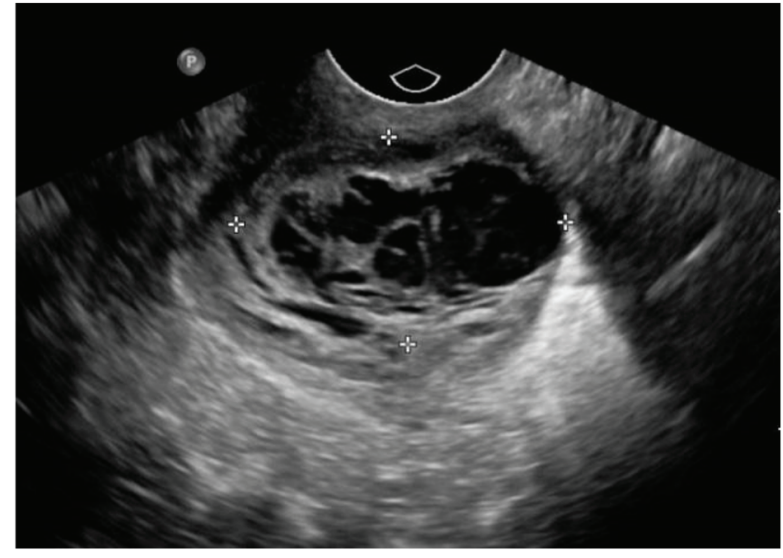

Figure 1. Ultrasound image showing a $4.2 \times 2.6 \mathrm{~cm}$ complex cystic structure containing multiple tubular serpentine cystic spaces in the lower anterior wall of the uterus close to the site of the previous LSCS scar.

contrast was seen.

Serial beta hCG was repeated $48 \mathrm{~h}$ later showing a suboptimal reduction to $513 \mathrm{IU} / \mathrm{L}$. In view of the elevated beta $\mathrm{hCG}$, a persistent scar ectopic pregnancy was considered a potential cause for the patient's vaginal bleeding with the possibility of concurrent AVM as evident on the ultrasound and MR images.

Patient was subsequently treated with intramuscular (IM) methotrexate for persistent elevation of serum beta hCG levels. She also underwent angiography as further evaluation of suspected uterine AVM. On angiography, uterine AVM was identified pre-dominantly supplied by the left uterine artery and early drainage via a large outflow vein was seen (Fig. 3a). The decision was made for UAE of the AVM. Left uterine artery was embolized using 355 - $500 \mathrm{~nm}$ PVA particles as it was technically unable to coil the AVM itself. There was also a tor- tuous but normal calibre left ovarian artery seen anastomosing to the supply in this region but this vessel was not embolized during this procedure to maintain collateral flow to the uterus. There was also a mass of tortuous vessels within the uterine corpus demonstrated on the right uterine arteriogram; this vessel was also embolized using the 355 - $500 \mathrm{~nm}$ PVA particles. Satisfactory devascularization of bilateral uterine arteries was achieved at the end of the procedure (Fig. 3b).

The patient was discharged home well 1 day after UAE. She was followed up in the out-patient clinic where her serum beta hCG levels down-trended to $48.8 \mathrm{IU} / \mathrm{L}$ by day 13 and to $9.6 \mathrm{IU} / \mathrm{L}$ by day 26 and was negative by day 58 after IM methotrexate treatment. She had two repeat pelvic ultrasound scans 3 and 7 weeks after UAE which showed cessation of blood flow to the AVM with interval reduction in size of posttreatment AVM. She also reported lighter periods at 2-month post-procedure follow-up visit.

\section{Discussion}

Uterine AVMs are considered rare and the true incidence of traumatic uterine AVMs is unknown. They may present with acute or chronic symptoms. Acute presentation could be a potentially life-threatening condition in which patients present with profuse uterine bleeding, possibly causing hemodynamic instability. Thus, it is an important differential to be considered in patients presenting with unexplained vaginal bleeding. Chronic features include recurrent abortions and poor pregnancy outcome [3].

Diagnosis of a uterine AVM should be based on pertinent patient history accompanied by negative beta $\mathrm{hCG}$ results and characteristic radiological findings. Angiography is the gold standard for the diagnosis of AVMs but it is rarely performed for diagnosis alone due to its invasive nature and is usually

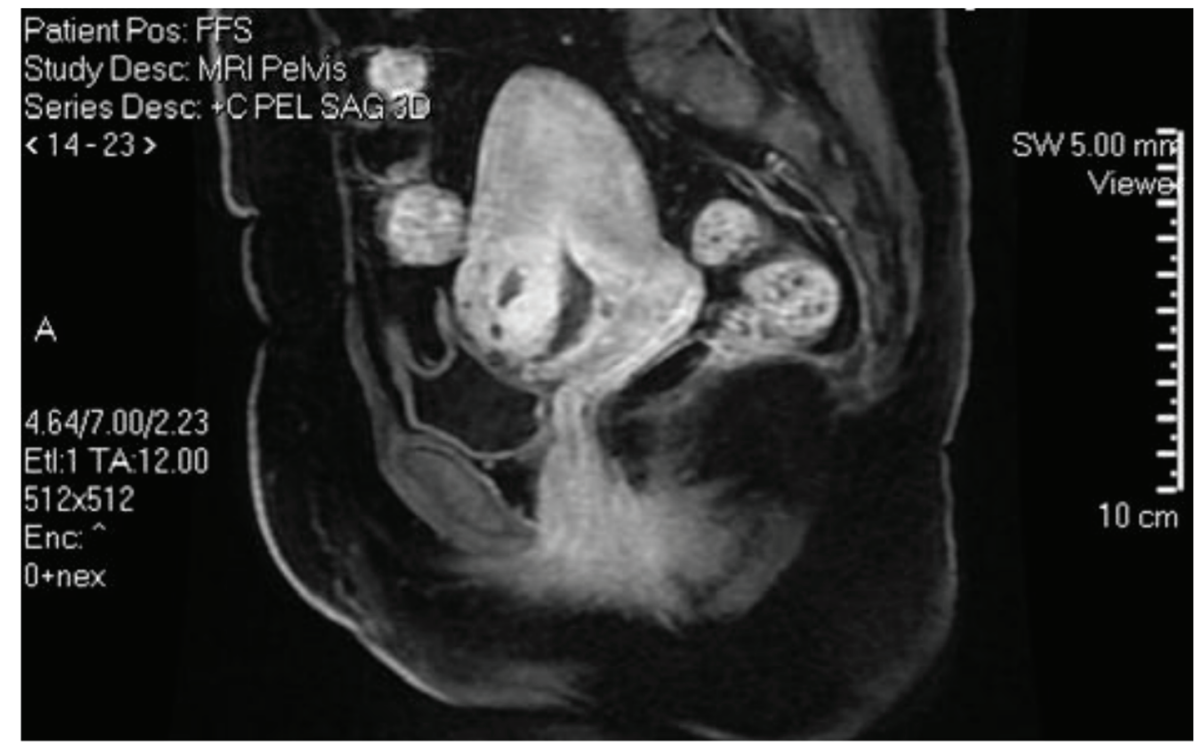

Figure 2. MRI showing $2.6 \mathrm{~cm}$ rounded, complex T2W hyperintense structure representing post dilation and curettage arteriovenous malformation. 

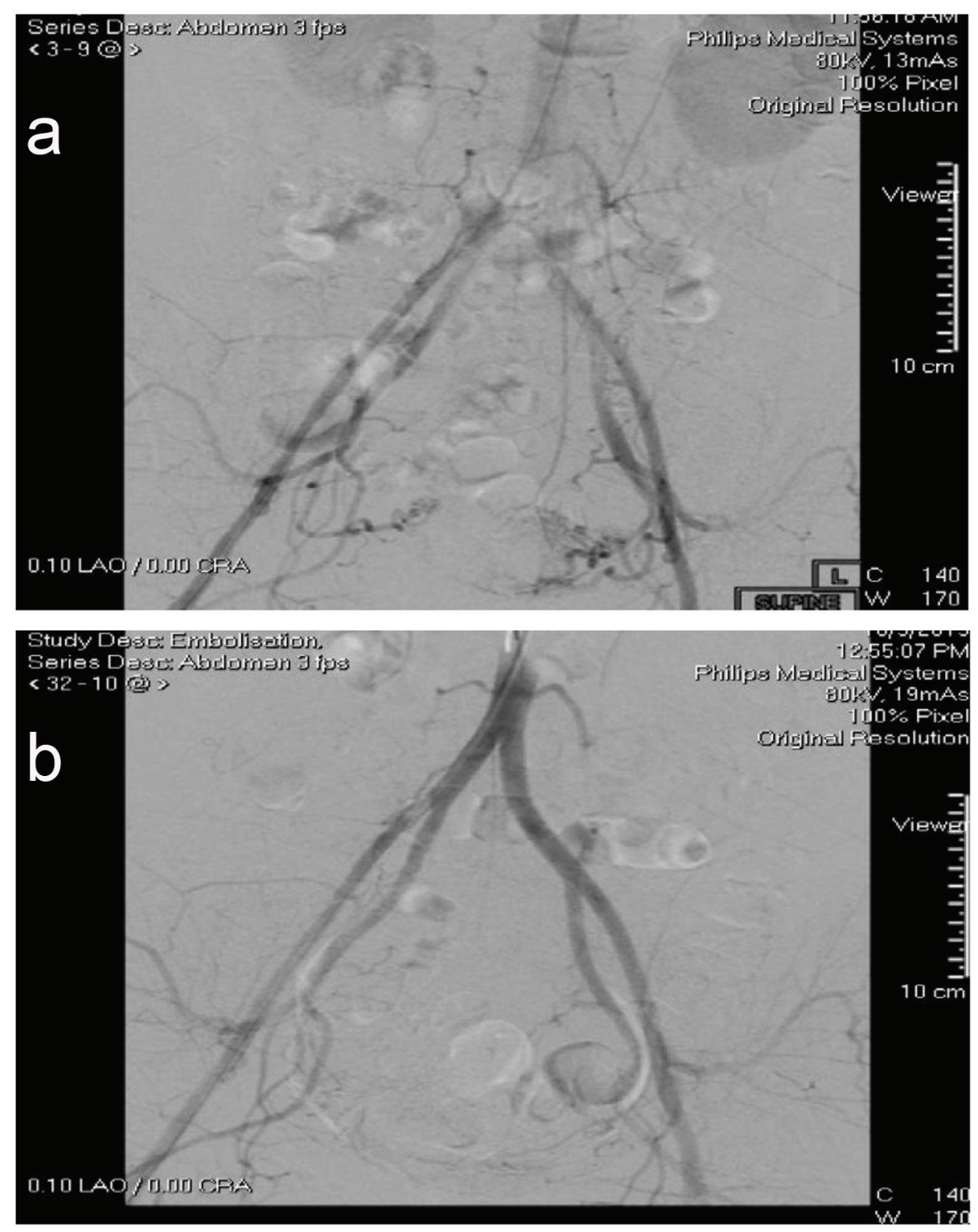

Figure 3. (a) Uterine AVM predominantly supplied by the left uterine artery is demonstrated and embolized using $355-500 \mathrm{~nm}$ PVA particles. (b) Satisfactory devascularization of bilateral uterine artery supply is documented post-procedure.

performed on patients requiring surgical intervention or embolization [4]. It shows typically an opacified vascular tangle with early venous filling. Angiography can also provide crucial information with regard to the vascular anatomy and the extent of the vascular malformation and this is important when performing interventional radiologic embolization [5].

Currently, most AVMs are diagnosed using less invasive methods such as ultrasonography or MRI. Transvaginal ultrasonography is commonly used for the initial evaluation of uterine AVM. In B mode, AVMs appear as heterogeneous, hypoechoic, indistinct tubular or cystic areas of variable size. Color Doppler shows tortuous vascular structures, with a multidirectional flow and apparent reverse flow due to aliasing. Duplex Doppler analysis shows low resistance flow with high velocity within the lesion [6].
On MRI, AVM typically manifests as multiple serpentine flow-related signal voids on T1 and T2-weighted sequences. Enhancement is similar to normal vessels. MRI is frequently necessary to identify the extent of the lesion [5].

Doppler and MRI features of uterine AVMs may overlap with other causes of arteriovenous shunting, including retained products of conception (RPOC), gestational trophoblastic disease, placental polyp, and vascular endometrial neoplasm [7]. Having a high index of suspicion for AVM among the differential diagnosis is crucial, as uterine curettage is indicated for retained products but contraindicated in AVMs as it can lead to catastrophic hemorrhage.

Past angiographic studies have demonstrated arteriovenous communication in $0.9 \%$ of normal uterine pregnancies, $20 \%$ of miscarriages and missed abortions, and $39 \%$ of ectopic 
pregnancies $[8,9]$. This pathophysiological shunting is thought to result from the development of arteriovenous fistulas within the placenta caused by necrosis of the chorionic villi. Consequently, this may explain why retained RPOC can mimic uterine AVM. Rufener et al [10] demonstrated that most of the uterine vascular abnormalities in postpartum and post-abortion patients initially thought to be uterine AVM at ultrasonography were ultimately found to be RPOC after histopathological evaluation. They suggested that serum beta hCG monitoring and follow-up ultrasonography or MRI might be useful for the diagnosis and treatment in these patients.

Our patient presented to us with a diagnostic dilemma as her clinical symptoms of persistent heavy vaginal bleeding could be either secondary to the AVM (as evident on both the ultrasound and MR images) or secondary to persistent scar ectopic pregnancy as she had elevated beta hCG levels, or have both conditions concurrently. RPOC as a differential diagnosis was possible but given that she had underwent a recent surgical uterine evacuation likely with secondary acquired AVM, a repeat dilation and curettage was not recommended.

Abnormal implantation in the cesarean scar bed accounts for $6 \%$ of ectopic pregnancies in multiparous women. More than half of these women have had one previous cesarean delivery $[11,12]$. AVMs have been confirmed as arising from a prior cesarean scar ectopic implantation site [11].

The contemporary management of uterine AVM is based on the symptomatology, the location, the extent, and the desire for future fertility. It is not necessary to treat uterine AVM in asymptomatic patients and conservative management should be considered as the primary approach, since most of these lesions tend to resolve spontaneously [13].

Effective treatment of uterine AVMs has also been reported using conservative medical management which may include therapy using danazol, prostaglandins, estrogens, methylergonovine [14] and even gonadotropin-releasing hormone agonist [15]. The potential mechanism which pharmacotherapy helps has not been elucidated, and has been thought to involve covering the bleeding vessels with proliferated endometrium facilitated by estrogen therapy and reduction in the blood flow to and collapse of the AVM using methylergonovine maleate [14].

Besides medical treatment, UAE has recently proved to be an effective alternative mode of treatment which is less invasive than surgery and has the benefit of fertility preservation [16]. Our patient was successfully treated with UAE using PVA particles. She fortunately did not require multiple sessions of embolization to bring her symptoms under control.

With experienced operators, UAE is generally safe. Minor complications include pelvic pain, hematoma, urinary tract infection, retention of urine, and vessel and nerve injury at the vascular puncture site. These require only mild supportive care or careful observation. Our patient also did not suffer from any severe or rare complications associated with UAE such as perineal skin sloughing, uterovaginal and recto-vesico-vaginal fistulae, or lower extremity neurological deficits [14, 17].

Finally, there is still a role for surgical management in cases where fertility has been completed or in cases where UAE has failed. The surgical options include uterine artery, internal iliac artery or ovarian ligament ligation and hysterectomy.

\section{Conclusion}

A high index of suspicion for acquired uterine AVMs should be reserved in the presence of risk factors such as recent uterine trauma from dilation and curettage. In our case, this may be complicated by a concurrent persistent scar ectopic pregnancy with diagnostic dilemma requiring a multi-disciplinary approach involving gynecologists and interventional radiologists. UAE is an effective alternative treatment of acquired AVMs after previous uterine surgery.

\section{References}

1. Chang KH, Park JK, Park SH, Kim HB, Park ST. Uterine arteriovenous malformation caused by intrauterine instrumentation for laparoscopic surgery due to left tubal pregnancy. Obstet Gynecol Sci. 2014;57(5):419-423.

2. Narang HK, Puri M, Patra S, Trivedi SS. Arterio-venous malformations of uterus - diagnostic and management dilemmas. J Obstet Gynaecol. 2015;35(6):632-637.

3. Rangarajan RD, Moloney JC, Anderson HJ. Diagnosis and nonsurgical management of uterine arteriovenous malformation. Cardiovasc Intervent Radiol. 2007;30(6):12671270.

4. Yazawa H, Soeda S, Hiraiwa T, Takaiwa M, HasegawaEndo S, Kojima M, Fujimori K. Prospective evaluation of the incidence of uterine vascular malformations developing after abortion or delivery. J Minim Invasive Gynecol. 2013;20(3):360-367.

5. Touhami O, Gregoire J, Noel P, Trinh XB, Plante M. Uterine arteriovenous malformations following gestational trophoblastic neoplasia: a systematic review. Eur J Obstet Gynecol Reprod Biol. 2014;181:54-59.

6. Levy-Zaubermann Y, Capmas P, Legendre G, Fernandez H. Laparoscopic management of uterine arteriovenous malformation via occlusion of internal iliac arteries. J Minim Invasive Gynecol. 2012;19(6):785-788.

7. Goyal S, Goyal A, Mahajan S, Sharma S, Dev G. Acquired uterine arteriovenous malformation developing in retained products of conception: a diagnostic dilemma. J Obstet Gynaecol Res. 2014;40(1):271-274.

8. Kido A, Togashi K, Koyama T, Ito H, Tatsumi K, Fujii S, Konishi J. Retained products of conception masquerading as acquired arteriovenous malformation. J Comput Assist Tomogr. 2003;27(1):88-92.

9. Jain K, Fogata M. Retained products of conception mimicking a large endometrial AVM: complete resolution following spontaneous abortion. J Clin Ultrasound. 2007;35(1):42-47.

10. Rufener SL, Adusumilli S, Weadock WJ, Caoili E. Sonography of uterine abnormalities in postpartum and postabortion patients: a potential pitfall of interpretation. J Ultrasound Med. 2008;27(3):343-348.

11. Moulder JK, Garrett LA, Salazar GM, Goodman A. The role of radical surgery in the management of acquired uterine arteriovenous malformation. Case Rep Oncol. $2013 ; 6(2): 303-310$. 
12. Rotas MA, Haberman S, Levgur M. Cesarean scar ectopic pregnancies: etiology, diagnosis, and management. Obstet Gynecol. 2006;107(6):1373-1381.

13. Dar P, Karmin I, Einstein MH. Arteriovenous malformations of the uterus: long-term follow-up. Gynecol Obstet Invest. 2008;66(3):157-161.

14. Molvi SN, Dash K, Rastogi H, Khanna SB. Transcatheter embolization of uterine arteriovenous malformation: report of 2 cases and review of literature. J Minim Invasive Gynecol. 2011;18(6):812-819.

15. Morikawa M, Yamada T, Yamada H, Minakami H. Effect of gonadotropin-releasing hormone agonist on a uterine arteriovenous malformation. Obstet Gynecol. 2006;108(3 Pt 2):751-753.

16. Milingos D, Doumplis D, Sieunarine K, Savage P, Lawson AD, Smith JR. Uterine arteriovenous malformation: fertility-sparing surgery using unilateral ligation of uterine artery and ovarian ligament. Int J Gynecol Cancer. 2007;17(3):735-737.

17. Garner EI, Meyerovitz M, Goldstein DP, Berkowitz RS. Successful term pregnancy after selective arterial embolization of symptomatic arteriovenous malformation in the setting of gestational trophoblastic tumor. Gynecol Oncol. 2003;88(1):69-72. 